\title{
Effects of Artifacts on the Diagnosis of Ultrasound Image
}

\author{
Alyaa Raheem \\ Assist Lecture, Middle Technical University / College of Medical \&Health Technology, Baghdad, Iraq
}

\begin{abstract}
In ultrasound imaging, the artifact is the term that can be used to describe various parts of an image that does not accurately represent the anatomic structure within the subject being evaluated. The current study aims to explain the foundation of understanding the artifacts in conventional ultrasound and Doppler techniques to highlight the effect which may occur as a result of these artifacts. Artifact in ultrasound imaging can be classified into three categories according to its cause, artifacts caused by the patient, by instrument, and by an operator. Recognition of artifacts is important as they can be considered as tissue composition indicators. Therefore, it helps in the diagnosis. The ability to recognize and correct potential ultrasound artifacts is significant for image quality improvement and optimal patient care. The most frequent artifact is acoustic shadowing that had been seen in 11 from 54 cases and the less frequent is blooming artifact that occurred in only 1 case from selected cases.
\end{abstract}

Keywords: artifacts, Doppler, image, ultrasound.

\section{Introduction}

The term artifact in radiography is used to define the portion of an image that does not represent real anatomical structures within the organs being evaluated. In ultrasound, artifacts are echoes that appear on the image but do not have a true correspondence to an anatomical structure. An artifact may cause the anatomical structure to be missing from the image. It also shows structures as present but incorrect in location, size, or brightness ${ }^{[1]}$. Ultrasound imaging uses sound waves to produce images from the inside of the body as considered safe, non-invasive, and does not use ionizing radiation. This procedure requires little to no special preparation ${ }^{[2]}$. Ultrasound is prone to numerous imaging artifacts, and these are commonly encountered in clinical practice. Artifacts have the potential to interfere with image interpretation. To avoid confusion, the radiologist should be able to recognize artifacts when they occur.

\section{Corresponding author:}

\section{Alyaa Raheem}

Email: aliamaan@yahoo.com.
Physicians should understand the physical explanation of the artifacts and use their knowledge to improve both the image quality of their scan and the diagnostic power of their interpretations. Ultrasound images can be created based on the physical properties of ultrasound pulse formation, the propagation of sound in the matter, the interaction of sound with reflective interfaces, echo detection, and processing. Ultrasound display equipment relies on physical assumption to assign the location and intensity of each received echo. This assumption to the echoes detected is originated from within the main ultrasound beam then the echo returns to the transducer after a single reflection [3]. The depth of an object is directly related to the time for an ultrasound pulse to return to the transducer as an echo and the frequency of the ultrasound, therefore, different transducers are used to scan diverse tissue. The speed of sound in human tissue is constant, the sound beam and its echo travel in a straight path, and the acoustic energy in an ultrasound field is uniformly attenuated ${ }^{[4]}$. In clinical Sonography, these assumptions are often not main maintained, when this occurs, echoes may be displayed erroneously and perceived as artifacts. Artifacts are arising either as a 
result of the errors of the ultrasound beam characteristics, the presence of several echo path velocity errors or as attenuation errors ${ }^{[5,6]}$.

\section{Artifacts in the conventional ultrasound.}

1. Artifacts caused by patients: these artifacts can be classified according to the following:

Artifacts from strongly reflective structures (shadowing): this artifact is mostly from gas, bone, stones of surgical clips, muscle does not reach sound well. Occurs when a sound beam attacks a highly reflective surface [see fig.1(a)]. In diagnostic large shadowing artifacts (acoustic dense structure) may obscure a deep pathologic process e.g., nodes. The shadowing can be made more obvious by increasing the frequency of the transducer. This artifact could be avoided by the sonographers. They may attempt to scan around gas or bone, change the angle to be oblique to the structure, and scans the areas below these structures. The artifact will be changed or disappear by this technique ${ }^{[7]}$.

Reverberation artifacts: Are most commonly seen adjacent to the bladder anterior wall, from the anterior surface wall make a simple cyst appear complex. Also, occur in the body in soft tissue as well as fluid, they may mimic mass. The reverberation pattern seen with bones is series of alternating lines. This type of artifact occurs when a large amount of sound is returned to the transducer because sound passes out of a structure with an acoustic impedance that is markedly different from its neighbor. ${ }^{[8]}$

Mirror artifacts: is the duplication of structure that occurs because the amount of sound returning may be so great that it is sent from the transducer back into tissues. It can appear when there is a curved structure; it may focus and reflect the sound like a mirror, most commonly when scanning the diaphragm. Theoretically, there should be no echoes from the lungs because they are full of gas, but in fact, there is a duplication of the structures within the liver above the diaphragm in all normal individuals. On the other hand, a mirror image can create a false impression of a pleural effusion because the diaphragm is also duplicated. This artifact occurs when the patient is scanned in an oblique axis in the coronal position. Lesion within the liver or spleen adjacent to the diaphragm can be duplicated in the lung. [9]

4.Enhancement effect: As the sound beam passes through fluid-filled structures or structure containing many cysts, it is not attenuated and there is an increase in the amplitude (brightness) of the echoes distal to the fluid. In the diagnostic a true pathologic condition may be obliterated by the increased gain distal to the fluidfilled structure. The acoustic enhancement may be useful in differentiating between solid and cystic lesions. ${ }^{[7]}$

5.Frezonal zone artifacts: occur in the field near the transducer that has little textural information in the first or two centimeters of the image. If there is a lesion lie close to the skin, it can be missed because much of the information in this area is noise.

6.Split - image artifact: The structure is duplicated because the refraction has occurred in the echoes that are returned to the transducer since it places at the assumed distance and direction. Mostly occur when the transducer is placed in the midline in the pelvis where the beam is bent at an oblique interface between tissues of different acoustic velocities from both sides of the muscles layer. When a double image is created, a single sac can be mistaken for a twin pregnancy. ${ }^{[8]}$

7.Slice-Thickness artifact: Occur when the relatively wide beam strikes the area between a fluid-filled "cyst" and soft tissue that is acutely angled. Low-level artifact echoes will be displayed within the fluid. In diagnostic low-level echoes in the posterior aspect of a cyst may be thought to be evidence of abnormal cyst contents. So can recognize it at the posterior aspect of the cyst and develop as the transducer moves from the center of the cyst. ${ }^{[9,11]}$

8.Comet effect: A dense echogenic line extending through the image occurs from a very strong acoustical interface, such as air bubbles, or metallic structure. The presence of this line may allow recognition of 
the metallic structure such as clips or loops within the uterus. ${ }^{[10]}$

9.Beam depth problem: artifacts occur in the far part of the field of the transducer beyond the focal zone. The echoes in this region are much coarser, and the major lesion may be missed (e.g., small metastases in the liver) if a long focus transducer is not used. Because considerable lateral beam spread occurs, the small pinpoint structure appears as transverse lines. ${ }^{[11]}$

10.Edge artifact: artifacts occur at the edge of a curved surface caused by scattering and refraction of sound waves. Occurs in the urinary bladder, cysts, and diaphragm if there is fluid on either side. ${ }^{[5]}$

\section{Artifacts caused by instrument}

1. Artifactual Noise: caused by electrical interference from nearby equipment. The noise produces a pattern (usually repetitive pattern) over the normal ultrasound image.

2.Main Bang artifact: caused by a strong interface appear like many echoes between the skin and the transducer, especially older transducer, lead to hiding subcutaneous and skin lesion. Also, this type may be seen because of poor technique or electronic focusing.

3.Grating lobes: caused by the periodic spacing of the phased array or, more commonly, linear array elements. Travel at an angle to the main beam. May appear as a septum that is present within an amniotic sac or another cystic process. The slightly curved septum is usually related to a strong curvilinear interface in the mid-portion of the linear array field. A curvilinear echo may be seen either at a shallower or deeper depth than the structure causing the artifact. ${ }^{[12]}$

4.Photographic artifacts: are a major problem, caused by undue brightness may also obscure subtle textural alterations because of the overall grayness of the image. Like dust on the camera, small echogenic areas will be seen on the camera image. If the echogenic mass lies within the liver, confusion with a metastatic lesion may occur.
5.Motion artifacts: occur in the structures that move rapidly, also may result from the exaggerated rapid motion of the transducer. Can recognize it when a wavy motion across the image is visible. Like fetal heart, it is not seen will infer that a fetus is dead.

6.Calibration problem: may not be clear on the image, but subsequent measurement using another ultrasonic system or phantom may show erroneous caliper measurement. Can be detected only by comparison with other system or by subtle measurement changes. In diagnostic, measurements such as the biparietal diameter may be wrong with tragic clinical consequences. ${ }^{[1]}$

7. Side Lobes artifact: are secondary echoes outside the main beam, that exist with all transducer. It is difficult to recognize unless quality control tests are performed. With at least several millimeters wide in the focal zone. Sidelobe artifact can be diminished by using the correct transmit focal zone and use the focusing system that comes with the transducer at the depth at which the artifact is greatest. [13]

\section{Artifacts caused by the operator (Technique)}

1. Operator scanning speed: are created when the scanning speed is too rapid, artifacts known as dropout lines are showed. To avoid this artifact, the most digital unit receives information rapidly. Some units appear to have gaps (the gaps are filled in with false echoes) between the lines of the image because they have not been 'smoothed". These little gaps between beams can eliminate in a cosmetic but uninformative fashion. ${ }^{[3]}$

2.Operator pressure artifacts: are created when too much or uneven pressure is applied while scanning. In diagnostic, scanning the fetal trunk using too much pressure with a linear array may make it appear to have a flattened ovoid shape rather than the preferred round shape. ${ }^{[11,14]}$

\section{Artifacts in Doppler ultrasound}

1. Aliasing: is the one most common artifact in Doppler ultrasound, seen both in color and spectral Doppler modalities. It reflects the point at which the 
maximum measurable velocity has been reached for a given pulse repetition frequency and gain setting. If the Doppler shift frequency exceeds half of pulse repetition frequency/Nyquist limits (normal is a $1-30 \mathrm{KHz}$ range), aliasing occurs and improper Doppler shift information (improper direction and value) results ${ }^{[15]}$.

2. Blooming Artifact: displays color outside of the vessel and makes vessels appear larger. This is usually caused by the gain setting, which can be adjusted. Decreasing the Doppler gain minimizes the blooming artifact, but the weakest signal from the smallest vessels may be undetected ${ }^{[16]}$.

3. Twinkling Artifact: is a color phenomenon seen behind a strongly reflecting interface such as urinary tract stones, parenchymal calcification, bones, or foreign bodies during the color Doppler ultrasound examination. This artifact appears as a color signal but without associated real flow ${ }^{[17,18]}$.
4. Spectral Mirror image: Appear due to divergence of the Doppler beam in two directions along the long axis of the vessel, since the ultrasound beam is perpendicular to the vessel. There will be the usurious impression of no flow (occlusion), or the flow direction may appear to be bi-direction. ${ }^{[19]}$

\section{Patients and Methods}

Fifty-four artifacts were studied in cases from October 2019 to February 2020, those cases were taken from Baghdad hospital (department of radiology), medical city, and 3 private clinics, done by using stationary ultrasound devices (Siemens, Philips \& SonoScape). The patients were conscious in the supine position. Ultrasound images that contained artifacts were classified according to the types of artifact, region of the exam such as (abdomen, brain, pelvic, chest, thyroid gland, liver, blood vessels) and causative agent. The study includes the common artifacts present in conventional and Doppler sonography that is used in medicine.

Table 1: Artifacts in conventional U/S

\begin{tabular}{|c|c|c|c|c|}
\hline Type of artifacts & Region & $\begin{array}{l}\text { Number } \\
\text { of cases }\end{array}$ & Rate & Causes \\
\hline \multirow[t]{4}{*}{ 1-Acoustic shadow } & Brain (infant) & 1 & $1.8 \%$ & Strongly reflected interface (bone) \\
\hline & Abdomen & 4 & $7.4 \%$ & Strongly reflected interface after gallstone \\
\hline & Pelvic & 4 & $7.4 \%$ & Strongly reflected interface from calcified mass. \\
\hline & Chest & 2 & $3.7 \%$ & Strongly refracted interface (Rib). \\
\hline \multirow[t]{4}{*}{ 2-Enhancement artifact } & Kidney & 2 & $3.7 \%$ & $\begin{array}{l}\text { Scattering and refraction(fluid) filled structure } \\
\text { after renal hydronephrosis }\end{array}$ \\
\hline & Breast & 2 & $3.7 \%$ & $\begin{array}{l}\text { Scattering and refraction (fluid) filled structure } \\
\text { from subcutaneous cyst }\end{array}$ \\
\hline & Abdomen & 2 & $3.7 \%$ & $\begin{array}{l}\text { Scattering and refraction (fluid) filled structure } \\
\text { after pleural effusion }\end{array}$ \\
\hline & Abdomen & 3 & $5.5 \%$ & $\begin{array}{l}\text { Scattering and refraction (fluid) filled structure } \\
\text { below abdominal ascites }\end{array}$ \\
\hline \multirow[t]{2}{*}{ 3- Mirror artifact } & Urinary bladder & 2 & $3.7 \%$ & $\begin{array}{l}\text { Extra reflection comes from the interface within } \\
\text { the body itself. }\end{array}$ \\
\hline & Liver & 1 & $1.8 \%$ & $\begin{array}{l}\text { Extra reflection comes from interface within the } \\
\text { body itself. }\end{array}$ \\
\hline
\end{tabular}


Cont... Table 1: Artifacts in conventional U/S

\begin{tabular}{|c|c|c|c|c|}
\hline 4-Noise artifact & Abdomen & 1 & $1.8 \%$ & Wrong time gain compensation T.G.C \\
\hline & Pelvic & 1 & $1.8 \%$ & Wrong time gain compensation T.G.C \\
\hline 5-Edge artifact & Breast & 2 & $3.7 \%$ & $\begin{array}{l}\text { Scattering and refraction at a tangential angle } \\
\text { from curved structure (mass) }\end{array}$ \\
\hline 6-Side lobe artifact & Urinary bladder & 2 & $3.7 \%$ & Sound reflected by adjacent structure. \\
\hline \multirow[t]{2}{*}{ 7-Comet-tail artifact } & Chest & 2 & $3.7 \%$ & $\begin{array}{c}\text { Collection of closely spaced perpendicular } \\
\text { echoes along one or two vectors in the U/S } \\
\text { image. }\end{array}$ \\
\hline & Abdomen & 2 & $3.7 \%$ & $\begin{array}{c}\text { Collection of closely spaced perpendicular } \\
\text { echoes along one or two vectors in the U/S } \\
\text { image }\end{array}$ \\
\hline $\begin{array}{l}\text { 8-Reverberation } \\
\text { artifact }\end{array}$ & Skin & 2 & $3.7 \%$ & Differences in acoustic impedance. \\
\hline $\begin{array}{l}\text { 9-Slice thickness } \\
\text { artifact }\end{array}$ & Urinary bladder & 3 & $5.5 \%$ & $\begin{array}{l}\text { When the boundary between the wall of the fluid } \\
\text { structure and its content is not perpendicular to } \\
\text { the interrogating sound beam. }\end{array}$ \\
\hline 10-Split image artifact & Pelvis & 2 & $3.7 \%$ & $\begin{array}{l}\text { The oblique interface between the tissues of } \\
\text { different acoustic velocities }\end{array}$ \\
\hline $\begin{array}{l}\text { 11-Motion artifact } \\
\text { (breathing) }\end{array}$ & Abdomen & 3 & $5.5 \%$ & Electrical interface from nearby equipment \\
\hline 12-Shadow artifact & Bowel & 1 & $1.8 \%$ & Less amount of gel \\
\hline
\end{tabular}

Table 2: Artifacts in the Doppler U/S

\begin{tabular}{|c|c|c|c|c|}
\hline $\begin{array}{c}\text { Type of artifact } \\
\text { 1-Color Aliasing }\end{array}$ & Region & $\begin{array}{c}\text { Number of } \\
\text { cases }\end{array}$ & Rate & Causes \\
\hline 2-Twinkle artifact & Carotid arteries & 3 & $5.5 \%$ & $\begin{array}{c}\text { Strongly reflected interface from hard plaque in } \\
\text { proximal ICA }\end{array}$ \\
\hline 3-Mirror artifact & Blood vessels & 1 & $1.8 \%$ & $\begin{array}{c}\text { Frequency exceed half of pulse repetition } \\
\text { frequency/Nyquist limit } \\
\text { directions along the long axis of vessels }\end{array}$ \\
\hline 4-Blooming artifact & Carotid arteries & 1 & $1.8 \%$ & $\begin{array}{c}\text { Increase in gain setting, extensive echogenic } \\
\text { because of irregularly surface plaque }\end{array}$ \\
\hline
\end{tabular}




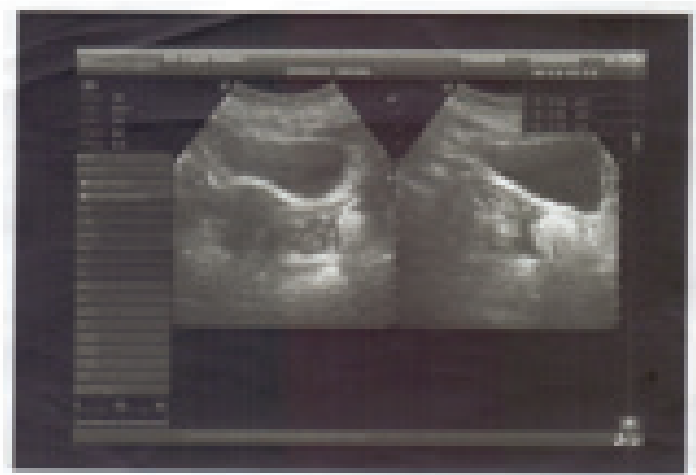

(a)

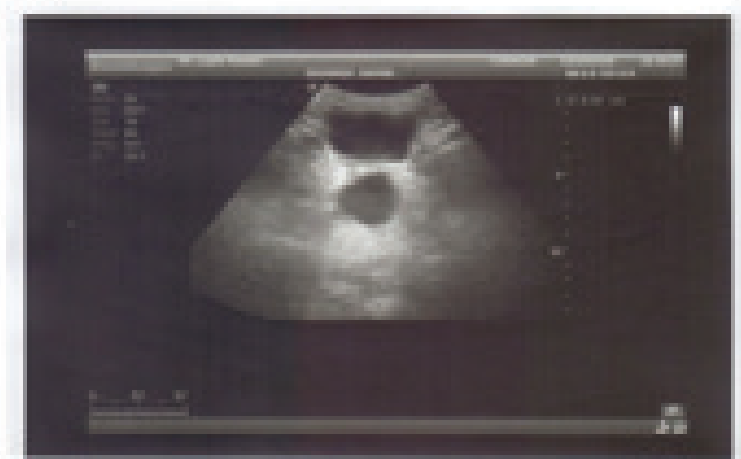

(c)
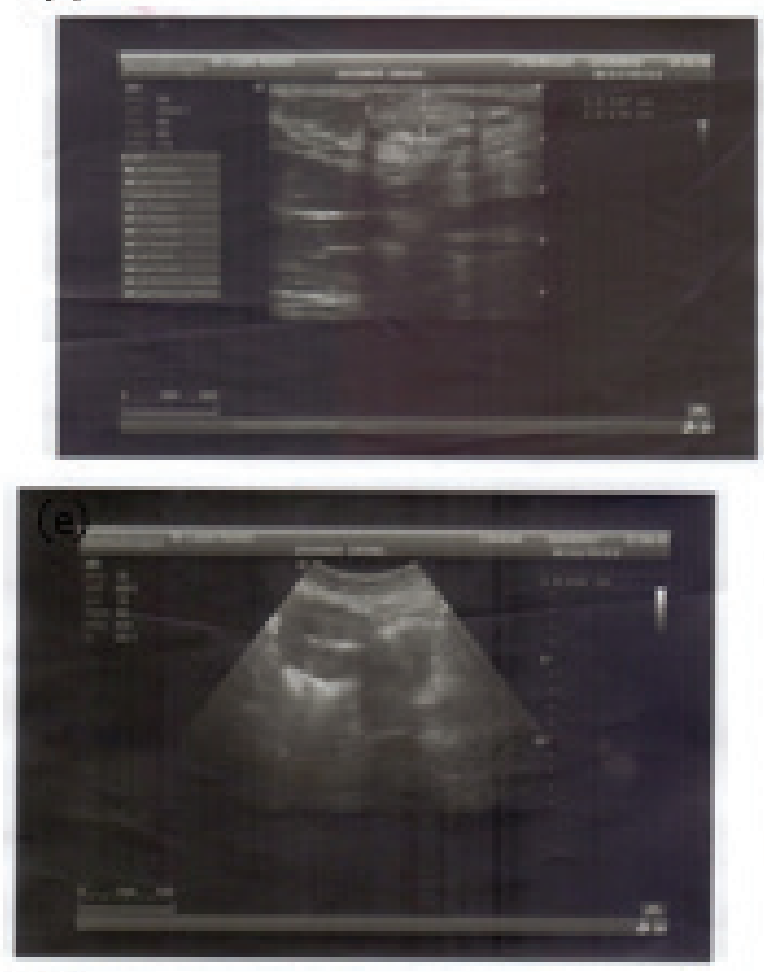

(E)

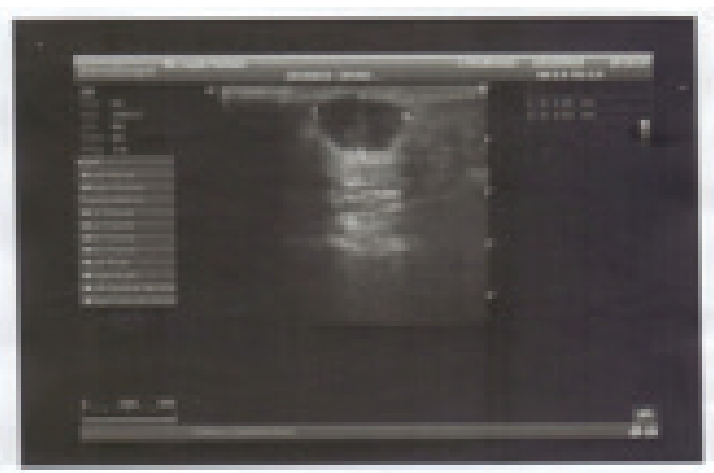

(b)

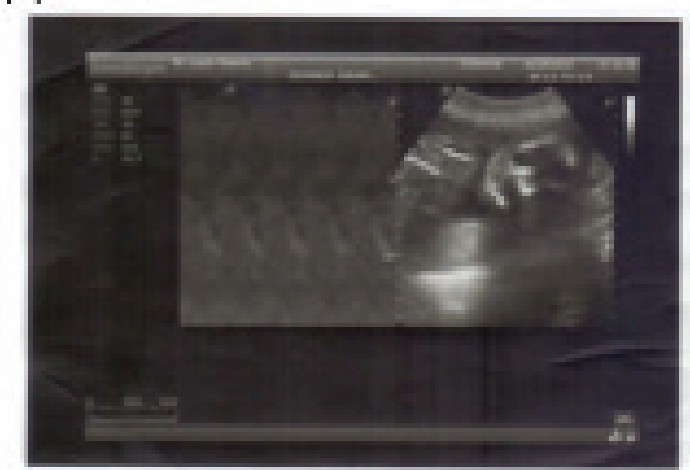

(d)
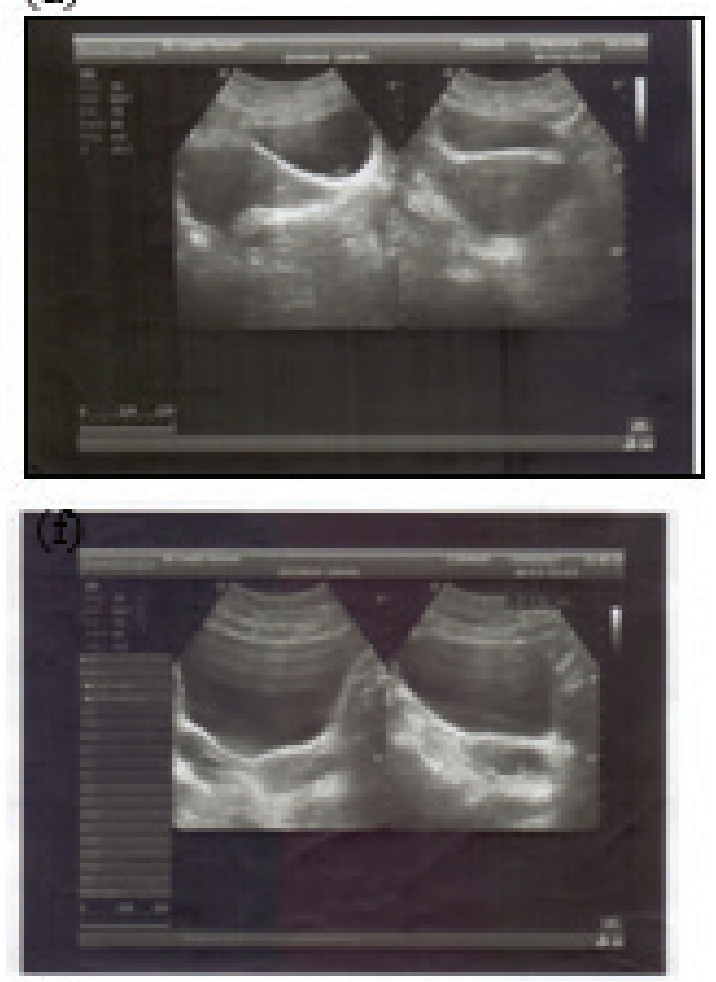

(h)

Fig.1. (a) Acoustic shadowing artifact from calcified mass, (b) Enhancement from subcutaneous cyst, (c) Mirror image of the bladder, (d) Noise artifact, (e) Edge artifact, from breast mass, (f) Sidelobe in the UB, (g) Comet tail from intrauterine device, (h) Reverberation artifact. 
(a)
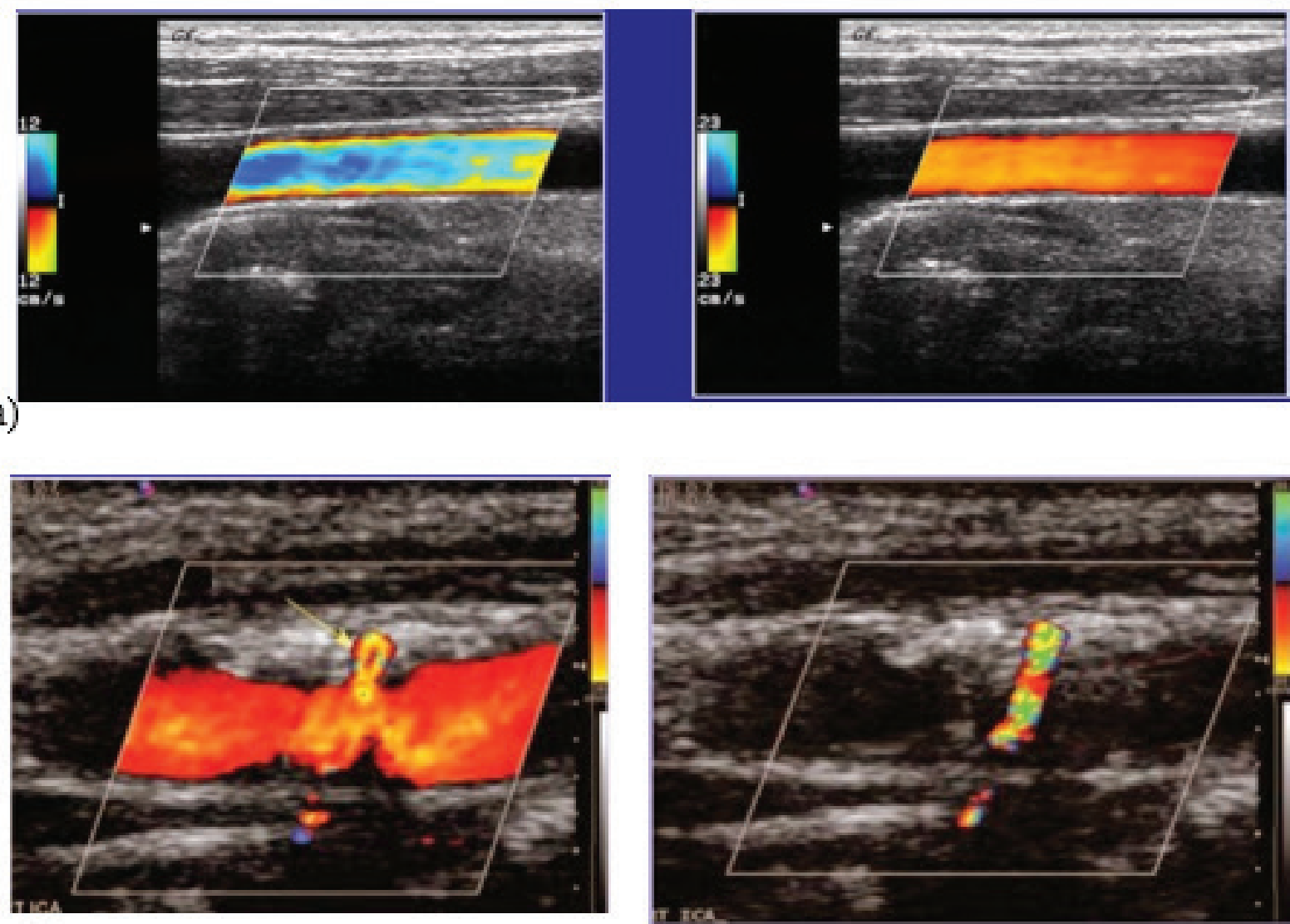

(b)

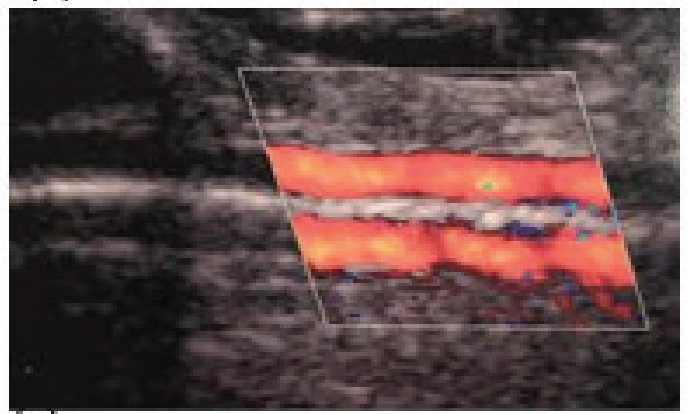

(c)

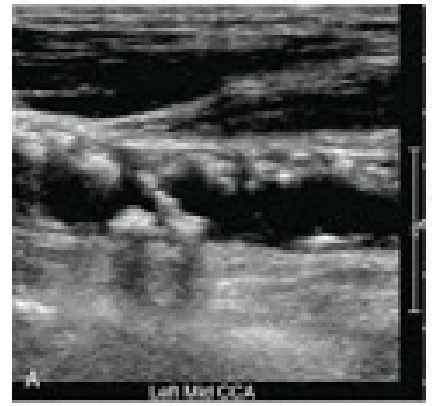

(d)

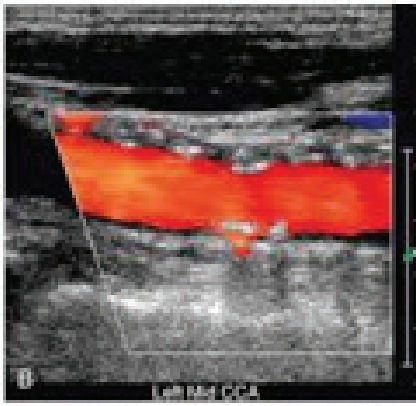

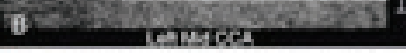

Fig.2, (a) color Doppler aliasing, (b)Twinkle artifact, Ulcerated plaque from hard plaque in the proximal ICA,(c) Mirror artifact of blood vessels, (d) Blooming artifact, extensive echogenic, irregularly surfaced plaque in the carotid on a longitudinal gray-scale.

\section{Discussion}

The most frequent artifact is Acoustic shadowing that had been seen in about 11 cases of 54. It appears like a dark or hypoechoic band deep to highly attenuating structures such as bone, gas, calculi, and calcification as shown in Fig 1(a). When sound strikes the interface, the sound beam is absorbed and reflected. This shadowing occurs when there is no sound conduction through the area and this can be very helpful in confirming renal and gall stone, also this shadowing can obscure pathologic processes. To avoid miss diagnosis change the PT position, the angle of the transducer, and the focal zone. The stone will be detected in all positions and the gases with be changed or disappear ${ }^{[1]}$. 
In the second place the enhancement artifact occurs in 9 cases, which appears as a hyperechoic area behind fluid-filled structures (e.g., gall bladder, urinary bladder, and cyst) as shown in Fig 1(b). this artifact is caused by decreased reflection. In the fluid, the sound waves attenuate less and are of a higher amplitude distally in comparison with an adjacent sound wave. This produces increased echogenicity that is seen as a bright band behind the fluid-filled structure. It is useful in differentiating between solid and cystic lesions. To correct this artifact, reduced the gain with spatial compounding different direction ${ }^{[9]}$.

Mirror artifact is detected in 3 images. It looks like duplicated equidistant from a strongly reflective interface. The return of the sound beam is delayed, and these delayed beams are reflected, displayed at a greater depth than their true anatomic depth (e.g., diaphragm, liver, and urinary bladder) as shown Fig1(c). To correct this artifact, scan from different angles, adjacent focal zone or TGC at the level of the diaphragm, scan from multiple windows ${ }^{[12]}$.

Noise artifact occur in about 2 images. This is an electrical interference artifact from nearby equipment (e.g., In an intensive care unit) as shown in Fig 1(d). Such noise has a repetitive pattern unlike the overall increase in the echogenicity seen with too much gain. To correct this type of artifact, the equipment can be modified to prevent such interference ${ }^{[13]}$.

Edge artifacts occur in about 2 from 54 cases. It appears as a shadow occurring at the edge of a curved surface (e.g., Cysts, urinary bladder, diaphragm if there is fluid on either side and mass) as shown in Fig1(e). Caused by sound waves encountering a curved surface at a tangential angle are scattered and refracted. This artifact disappears when changing the angle of scanning [5].

Sidelobe artifacts appeared in two cases. It looks as a hyperechoic rounded object with an anechoic or hypoechoic structure (e.g., Urinary bladder, gall bladder, and needle biopsy) as shown in Fig1(f). In a linear array transducer, multiple other low-amplitude beams project radially at different angles away from the main-beam axis, these are termed side lobes. It can be reduced by using very closely spaced elements in the array (focusing system that comes with the transducer at the depth at which artifact is greatest) or using transmit focal zone ${ }^{[18]}$.

four cases of Comet-tail artifact were recognized. It appears as series of multiple, closely spaced small bands of echoes from reflective interfaces and thus sequential echoes are closely spaced that individual signals are not perceivable in the image (e.g., Surgical clips, copper intrauterine device) as shown in Fig1(g). To correct this artifact, decrease TGC near the near gain, change beam angle, and alternative window ${ }^{[11]}$.

Reverberation artifact is noticed in two images. It appears as a multiple equidistantly spaced linearlike ladder as shown in Fig 1(h). It is generated from a primary U/S beam which may repeatedly cause a parallel highly reflective surface. The echoes reflected back and forth before returning to the transducer for detection. This artifact can be prevented by decrease TGC near in the near gain, change beam angle in an alternative window ${ }^{[10]}$.

Slice thickness artifact is detected in only 3 cases. It seems to be as false sludge or debris within anechoic cystic structure close to the distal wall due to the wide beamwidth. It is caused when the boundary between the wall of a cyst, gall bladder, or urinary bladder and the containing fluid is not perpendicular to the interrogating sound beam. To prevent this artifact, change the focal zone or scan from different angle ${ }^{[11,14]}$

Split image artifact occurs in 2 cases. This artifact is also called a refraction artifact. It is the bending of the sound beam at an oblique interface between tissues of different acoustic velocities. This artifact is the displacement of the distal structure by more proximal refraction of the beam. Refraction can also displace the position of a deeper interface such as the diaphragm deep to a small cyst in the liver, since the beam travel more slowly in fluid than in the liver, a large cyst can delay the passage of sound causing the diaphragm to appear 
further away than it is. A small cyst can cause a peculiar effect on the diaphragm. This artifact can be eliminated if the transducer is moved to one side or the other ${ }^{[20]}$.

Motion artifact (breathing) occurs in about 3 cases. If the patient breathes while scanning the image may be distorted and blurred because a part of the scan will be performed during inspiration and part during expiration. Also, this artifact may occur due to differences in the focal zone during respiration. To correct this artifact, ask the patient to hold his breath, or utilize the cine loop control to review the last frames of the scan and freeze when the most desirable image appears. If a patient is unable to hold his breath, make sure the persistence is set low and that simultaneous multiple electronic focusing is not slowing the frame rate. These techniques will shorten the time to stabilize the image before it can be frozen ${ }^{[13]}$.

Color Doppler aliasing artifacts occur in 5 cases. Aliasing appears due to an insufficient sampling rate and occurs when the frequency shift to be measured is more than twice the pulse repetition frequency as shown in Fig 2(a). The artifact result in a wrap round of Doppler spectrum in pulsed or color Doppler ultrasound. [21] Aliasing at pulsed Doppler appears as a folding over of forwarding flow in systole in the reverse direction. Aliasing at color Doppler ultrasound may manifest as a mixture of colors or as a focus of color in the vessel corresponding to a continuum of colors folded over from normal flow in the opposite direction. This artifact can be useful for detecting of stenosis. It can be reduced by increasing the pulse repetition frequency or using a lower frequency transducer, thus decreasing the Doppler shift ${ }^{[23]}$.

Twinkle artifact appears in 3 scanning. Is the result of intrinsic machine noise seen with color Doppler ultrasound as shown in Fig2(b). It occurs as a focus of alternating colors on Doppler signal behind the reflective object. Twinkle artifact is more sensitive for detection of small stone (e.g., Urolithiasis) than is acoustic shadowing, and is highly dependent on machine setting, and is most pronounced when the reflecting surface is rough. The way to correct this artifact, change the focal zone since the twinkle artifact becomes more obvious when the focal zone is located below a rough reflecting surface than when it is above it ${ }^{[20]}$.

Blooming artifact occurs in just 1 image of the collected cases. It makes vessels appear larger because it displays color outside the vessels as shown in Fig 2(d). To minimize this artifact, the operator should reduce the gain ${ }^{[19,22]}$.

\section{Conclusion}

Despite the continued improvement and sophistication of ultrasound technology, artifacts have not disappeared. Some of the newer transducer shapes and geometry can cause increased artifact appearances which are helpful in certain circumstances. If an artifact appears in the wrong place at the wrong time, miss diagnoses can be made, appropriate machine setting can eliminate only some artifacts. The best defense against misinterpretation of artifacts relies on the educating procedures of the sonographer. The operator should study all the types of artifacts, concentrate and take enough time to set the instrument and scan from deferent windows to distinguish between artifact and real pathology.

\section{References}

1- Feldman, M.K., Katyal, S. \& Blackwood, M.S. US artifacts. Radiographics : a review publication of the Radiological Society of North America, Inc, 2009. 29(4), pp.1179-89.

2- Cosgrove, David. Manual of Diagnostic Ultrasound, Volume 1 (Second Edition). Ultrasound in Medicine \& Biology. 2012. 38. 1848.10.1016/j. ultrasmedbio.2012.05.021.

3- Wells, P.N.T. Ultrasonic imaging of the human body. Reports on Progress in Physics, 1999. 62(5), pp.671-722.

4- Tole, N.M. Basic Physics of Ultrasonographic Imaging. , pp.1-5. Essential Health Technologies Health Technology and Pharmaceuticals, 2005. ISBN 9241592990 (NLM classification: WN 208).

5- Hindi, A., Peterson, C. and Barr, R. G. 'Reports in Medical Imaging Artifacts in diagnostic 
ultrasound', Reports in Medical Imaging, 2013.pp. 6-29. doi: 10.2147/RMI.S33464.

6- Palmer, Philip E. S, Breyer, B, Bruguera, C. A, Gharbi, H. A, Goldberg, B. B. et al. Manual of diagnostic ultrasound / edited by P. E. S. Palmer. Geneva : World Health Organization. [1995ן. ISBN: 9241544619. 5225035949 (Russian).'

7- Faulkner K. Physics for diagnostic radiology. 3rd edition. $\mathrm{Br} \quad J \quad$ Radiol. 2012;85(1011):290. doi:10.1259/bjr/89098047.

8- Pineda VC, Macías PM, Bernal GA. Basic physical principles of ultrasound. Investigación en Discapacidad .2012;1(1):25-34.

9- Sutton, David. "A textbook of radiology and imaging.' ed, New York: Churchill Livingstone, 1987, 1,857 pp. 250.00 Journal of Nuclear Medicine May (1990), 31 (5) 702-703.

10- Fulton R. Focused-Basic Ultrasound Principles and Artifacts, Focused Ultrasound Techniques for the Small Animal Practitioner. 2014. First Edition. Published 2014 by John Wiley \& Sons.

11- Goldstein, Albert, and Beatrice L. Madrazo. "Slice-thickness artifacts in gray-scale ultrasound." Journal of Clinical Ultrasound .1981. 9.7: 365-375.

12- Laing, F. C. "Commonly encountered artifacts in clinical ultrasound." Semin Ultrasound 1983. 4.1: 27-43.

13- Muller, N., et al. "Ultrasonic refraction by the rectus abdominis muscles: the double image artifact. " Journal of ultrasound in medicine, 1984. 3. 11: 515-519.
14- Morley, Patricia, Gabriel Donald, and Roger C. Sanders, eds. Ultrasonic sectional anatomy. Elsevier. 2013.

15- Teh, James. "Applications of Doppler imaging in the musculoskeletal system." Current problems in diagnostic radiology.2006. 35.1: 22-34.

16- Boesen, M. I., et al. "Musculoskeletal colour/power Doppler in sports medicine: image parameters, artefacts, image interpretation and therapy." Clinical and experimental rheumatology 28.1,2009: 103 113.

17- Nilsson, Anders. "Artefacts in sonography and Doppler.” European radiology. 2001. 11.8: 1308.

18- Rahmouni, Alain, et al."Color Doppler twinkling artifact in hyperechoic regions." Radiology.1996.199.1: 269-271.

19- Reading, C. C., et al, "Color and spectral Doppler mirror-image artifact of the subclavian artery ." Radiology ,1990,174. 1: 41-42.

20- Middleton, W. D., and G. L. Melson. 'The carotid ghost. A color Doppler ultrasound duplication artifact." Journal of ultrasound in medicine 1990. 9.9: 487-493.

21- Lee, Jae Young, et al. 'Color and power Doppler twinkling artifacts from urinary stones: clinical observations and phantom studies. 'American Journal of Roentgenology. .2001.176.6: 14411445 .

22- John Pellerito Joseph Pola. Introduction to vascular ultrasonography. Philadelphia: Elsevier Saunders. 2012. 6 ed, eBook ISBN: 9781455737666

23- Kenneth J. W. Taylor, Peter N. Burns and Peter N. T. Wells. "Clinical applications of Doppler ultrasound.1995. 415 pp. New York: Raven. 